\title{
Partenariats d'éducation au patrimoine entre professeurs et intervenants d'un parc naturel régional : de la compétence d'un collectif à une compétence collective
}

\author{
Heritage education partnerships between teachers and stakeholders in \\ a regional natural park: from the competence of a collective to a \\ collective competence
}

\author{
Sylviane Blanc-Maximin ${ }^{1}$ \\ ${ }^{1}$ Université d'Aix-Marseille. Laboratoire ADEF. sylviane.maximin@univ-amu.fr
}

RÉSUMÉ. A l'école primaire française, la modalité pédagogique constituée de projets partenariaux avec les parcs naturels régionaux permet une rencontre entre les éducations formelle et non formelle au patrimoine. Axé sur l'étude des pratiques partenariales des acteurs éducatifs (enseignants et intervenants d'un parc naturel régional), nous cherchons à comprendre dans quelle mesure et sous quelles conditions celles-ci peuvent déboucher sur la mobilisation d'une compétence professionnelles de nature collective. Trois études de cas ont été outillées méthodologiquement par une analyse de contenu des projets, des observations et captations vidéo lors d'entretiens entre les partenaires et de séances de classe. Les résultats permettent d'identifier des niveaux de partenariat très différents qui offrent ou non des opportunités de développement professionnel. Les obstacles repérés se situent majoritairement au niveau des visées des acteurs éducatifs dans ces projets d'éducation au patrimoine.

ABSTRACT. In French primary schools, partnership projects with regional natural park stakeholders allow us to reconcile formal and informal heritage education approaches. Based on partnership practices of educational actors (teachers and regional natural park workers) we seek to understand to what extent and under which conditions these could lead to the acquisition of professional skills of a collective nature. In three case studies, a methodology framework was used to analyse the projects, observations and video recordings picked up during exchanges between partners and in sessions with the class. The results allow us to identify very different levels of partnership that offer or not opportunities for professional development. The obstacles rest mainly the ambitions of educational actors in these heritage education projects.

MOTS-CLES. Partenariat éducatif, compétence collective, compétence d'un collectif, territoire local, éducation au patrimoine.

KEYWORDS. Educational partnership, community skill, skill of a community, local area, heritage education.

\section{Introduction : partenariat éducatif et territoire local}

Une dynamique territoriale se concrétise actuellement dans une inflation de projets partenariaux dont l'École est fréquemment partie prenante. Dans une logique de déconcentration, un partenariat a été scellé dans une convention signée entre les Parcs Naturels Régionaux (PNR) et le Ministère de l'Éducation Nationale. Elle se décline dans chaque parc sous la forme d'une convention ratifiée par le PNR concerné et la circonscription de l'Éducation Nationale présente sur le territoire. Ce lien établi entre les établissements scolaires et les acteurs des territoires occasionne une rencontre entre une éducation formelle au patrimoine et une éducation non formelle. Cette rencontre est identifiée comme une évolution des rapports aux savoirs (Charlot, 1997 ; Khalali et al., 2019) vers l'expérientiel (Berryman, 2007) qui est revendiqué comme démarche privilégiée par les PNR. Les acquisitions et les connaissances acquises sur le terrain hors de la classe, entraînent une modification partielle de la forme scolaire (Vincent et al, 1994; Maulini et Perrenoud, 2005). Elles sont susceptibles de modifier les rapports aux savoirs (Khalali et al, ibid) car l'expérience « vécue » 


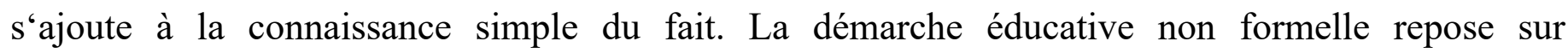
l'interaction et le contact direct entre l'apprenant et son territoire qui permettrait d'acquérir des savoir-faire et des connaissances contextualisées. L'éducation formelle est définie, quant à elle, comme « espace métaphorique de rencontre entre l'élève et la source des savoirs, entre le récepteur et l'émetteur des contenus de formation, dans une relation nouvelle, construite pour dire le sens des savoirs (Garnier, 2014).

Nous ne développerons pas ici les questions épistémologiques posées au sujet de la forme scolaire. Notre étude est orientée sur les pratiques partenariales entre enseignants et intervenants d'un PNR initiées dans des projets d'éducation au patrimoine. Afin de bien saisir les enjeux présents et leurs impacts dans l'activité éducative, il est indispensable de décrire les contextes professionnels de chaque partenaire et les valeurs qu'ils sont censés incarner et/ou transmettre.

Les objectifs actuels que vise l'ensemble des parcs naturels régionaux (PNR) français se déclinent ainsi : innover en faveur du développement durable, valoriser le patrimoine, soutenir l'économie locale et éduquer à l'environnement (source : site officiel des Parcs naturels régionaux de France). Un PNR œuvre donc dans une synergie économique sociale et éducative sur une aire protégée. Le patrimoine occupe une place importante puisqu'il est question de le valoriser. Cette valorisation comme dans les territoires et aires protégées s'organise au moins sur deux axes : une économie touristique et une dynamique éducative. Cette démarche est portée par des valeurs précisées dans des chartes. Par exemple, celle de la région Provence-Alpes-Côte d'Azur (PACA) s'appuie sur un principe de mutualisation des savoirs et des compétences afin d'enrichir les pratiques et nourrir la qualité des projets, l'innovation et la créativité selon le fichier ETT Charte éducation territoire des PNR de PACA. «Le Parc travaille avec les acteurs du territoire qui se reconnaissent dans les principes éducatifs qu'il défend. Il veille à ce que les projets conduits s'inscrivent dans la durée, et privilégient les démarches pédagogiques actives. » (Ibid, 2012).

Concrètement, un PNR dispose d'un carnet d'adresses d'acteurs éducatifs souvent autoentrepreneurs aux compétences diverses selon les régions (par exemple : accompagnateur moyenne montagne, botaniste, guide pêche, potier, musicien ...) et d'associations partenaires (associations de protection du patrimoine, de randonnée pédestre, ...) qu'il sollicite en fonction de propositions de projets pédagogiques d'enseignants du premier et du second degré. Les intervenants ne sont ni fonctionnaires ni recrutés avec un contrat de travail à durée indéterminée. Leur statut se rapproche de celui d'un intermittent (rémunéré «à la tâche »). Un PNR les sollicite en fonction des besoins exprimés par les enseignants qui souhaitent engager leurs classes dans des projets partenariaux.

Du côté de l'Éducation Nationale, les professeurs sont invités par leur institution à élaborer des pré-projets éducatifs qui préexistent à une rencontre entre les futurs partenaires. Dans ce cadre, les contenus permettent au PNR de solliciter les intervenants identifiés comme des experts dans un domaine. Les enseignants sont porteurs de compétences professionnelles inscrites dans un référentiel métier (M.E.N., 2013) et notamment celle de « coopérer avec les partenaires de l'école ». Un autre texte institutionnel - la circulaire ${ }^{\circ} 92-196$ du 3 juillet 1992 participation d'intervenants extérieurs aux activités d'enseignement dans les écoles maternelles et élémentaires- décrit explicitement les raisons qui préfigurent la présence d'intervenants dans le cadre scolaire formel. Elle est légitimée par une expertise dans un domaine précis qui fait défaut au professeur. La présence d'un intervenant extérieur y remédie ce qui n'est pas sans poser des questions d'identité professionnelle du côté des enseignants.

Pour bien saisir les enjeux du partenariat, nous allons préciser ce concept et les éléments saillants de sa mise en pratique. Nous appréhenderons ce phénomène du côté du travail conjoint partenaireenseignant qui engage des positionnements professionnels spécifiques. Au lieu de le penser en termes de manques (notamment du côté enseignant), nous chercherons à savoir si ces situations de 
rencontres professionnelles peuvent être l'occasion d'enrichir d'une manière spécifique, par une mobilisation de compétences de nature collective, les professionnels.

\section{Le partenariat éducatif}

En 2006, Mérini a mis au jour les attentes suscitées par une situation partenariale : l'ouverture à l'autre, la dimension du réel et l'authenticité de la mise en oeuvre pratique dans un système d'apprentissage (celui de l'école) plus coutumièrement tourné vers un savoir jugé savant et " scolarisé », sous l'influence de la didactique. Dans le cas de l'éducation au patrimoine en lien avec le territoire, les enseignants se positionnent dans une "orientation vers une école "intégrée » sur son territoire ouverte aux parties prenantes territoriales »(Lange, 2017, p.82). Celle-ci dépasse l'orientation d'une école ouverte sur le territoire, guidée par l'enseignant seul, comme cela était le cas par exemple, dans la classe-promenade initiée par Célestin Freinet.

L'intervention d'un acteur extérieur au monde scolaire positionné comme un expert devant combler les manques de l'enseignant soulève à la fois enrichissement pour tous (élèves et enseignant éducateur) et tensions. En effet, cette modalité de travail s'inscrit dans la double culture éducative présente entre pratiques scolaires et pratiques sociales de référence qui existe par exemple dans l'éducation au patrimoine. La richesse et la difficulté du travail partenarial proviennent d'une interaction entre des valeurs propres à chacun dans une altération (Ardoino, 1993) des deux cultures qui conduisent Clanet (1990) à développer le concept d'interculturalité. La situation partenariale est potentiellement basée sur une interaction riche entre les acteurs, sur la mise au jour de nouvelles valeurs professionnelles, de regards croisés sur des thématiques complexes avec des méthodes différentes. Mais cette interaction est délicate car elle peut engendrer des conflits inter et intra psychiques, d'identités professionnelles qui appellent des négociations et des renormalisations chez les acteurs (Schwartz et Durrive, 2003). En effet, dans cette rencontre interculturelle entre professionnels, l'étude INRP de 1994 dirigée par Zay identifie la présence d'enjeux forts pour les enseignants: disciplinaires, idéologiques, d'identité professionnelle (« L'enseignant ne peut plus se définir par la connaissance des contenus car il attend de sa collaboration avec l'autre, que celui-ci lui apporte plus que ce qu'il en sait. »), de modification de pratiques pédagogiques, de modalités d'évaluation, d'auto-formation ou de formation mutuelle (Eneau, 2016; Dupont, 2010). Cette formation peut prendre la forme de compétences professionnelles. Une compétence de négociation peut viser l'acquisition entre les professionnels. La négociation concerne prioritairement l'aspect programmatique (Boutinet, 2005) du projet : montage financier, planification et durée des actions, choix du produit fini précisés par un ensemble d'objectifs à atteindre lors de tâches ou de situations identifiées. Thomazet et Mérini (2014) affirment que c'est sans doute ici qu'intervient de manière déterminante la question du partenariat, telle que nous avons pu la définir, c'est-à-dire une action négociée prenant en compte les intérêts de chacun dans la perspective d'une plus grande efficacité sur le parcours scolaire de l'élève (Mérini, 2007). Cette action suppose des compétences en matière de négociation, de contractualisation, et dans la régulation de la prise de décision. Mais ce travail d'architecture laisse souvent dans l'ombre une discussion sur la dimension du processus éducatif présent dans un projet (Boutinet, ibid). En effet, dans les trois axes majeurs qui semblent fonder les pratiques collaboratives (Thomazet et Mérini, 2014), les deux axes d'élaboration, de planification de l'action et de la communication occultent souvent le troisième axe, celui de l'évaluation, qui participe justement du processus d'apprentissage et du développement professionnel des éducateurs et des enseignants. En effet, « les métiers ne sont pas monolithiques et ne sont pas le seul résultat d'une prescription qu'elle soit réglementaire (prescription primaire) ou liée à la formation (prescription secondaire). Les professionnels inventent et réinventent leur métier : «L'homme est plein à chaque minute de possibilités non réalisées » (Vygotski, 1925/1994) car l'activité réalisée et l'activité réelle ne se recoupent pas (Clot, 2007). Le repérage des différentes configurations de pratiques collaboratives (Thomazet et Mérini, ibid) débouche sur une typologie : pratiques 
partenariales, échanges collaboratifs opérationnalisant des décisions (autocratiques, de soustraitance, autres) échanges collaboratifs de médiation et pratiques conjointes.

\subsection{Le partenariat dans l'Éducation Nationale}

La notion de partenariat apparaît en France dans la loi d'orientation de 1990 qui introduit la notion de compétence dans le domaine de l'Éducation Nationale. En effet, la logique de compétences (Zarifian, 1999) issue du monde l'entreprise étant transposée dans le cadre scolaire depuis 1991, engage les professeurs à mobiliser des compétences professionnelles dans l'exercice de leur métier. A ce titre, un référentiel de compétences professionnelles régit la professionnalisation des enseignants dans lequel le professeur doit «coopérer avec les partenaires de l'école » et notamment avec « les structures culturelles et les acteurs socioéconomiques, en identifiant le rôle et l'action de chacun de ces partenaires » $(M E N, 2013)$. Le partenariat à l'école vise à introduire la présence de partenaires experts dans un domaine précis dans le système d'apprentissage habituel de l'école. Au stade des pratiques, il sollicite différentes postures de nature collective : coordination, collaboration, coopération (Marcel, 2007) chez l'enseignant et chez l'intervenant.

Le partenariat dans un projet d' « éducation à » en lien avec un territoire fait appel à plusieurs de ces configurations. Il nécessite chez les acteurs éducatifs des compétences de négociation, de contractualisation, de régulation pour une prise de décision commune et dans la mise en œuvre concrète. C'est pourquoi les concepts d'intelligence collective (Lévy, 1994, 2003) et de compétence collective [Wittorski (1997) ; Dubois et Retour (1999) ; Astier (2006) ; (Blanc-Maximin et Eymard (2010)] bâtis initialement sur une interdépendance de spécialistes, d'experts devraient pouvoir bénéficier à des «éducations à » (comme l'éducation au patrimoine) dans des projets de territoire. En effet, dans le cadre actuel des programmes d'enseignement, celles-ci sont positionnées comme des îlots rationnels d'interdisciplinarité (Fourez, 1998). De plus, dans le cadre du territoire, les « éducations à » sollicitent des compétences professionnelles chez les intervenants PNR (membres d'associations, d'aires protégées ...) qui bousculent les professionnalités enseignantes et plus largement questionnent le rôle de l'école et les apprentissages qui y sont réalisés. Le travail partenarial est non seulement confronté à des rencontres de savoirs entre acteurs mais il est affecté par des conflits de valeurs. Celles dont sont porteurs les acteurs mais aussi celles de l'éducation au patrimoine qui véhicule des valeurs contradictoires (Barthes, Alpe, Blanc-Maximin, 2015). Si ces tensions engendrent des conflits, ceux-ci peuvent aussi déboucher sur la mobilisation chez les acteurs d'une compétence de nature collective.

\subsection{Un partenariat créateur de compétences professionnelles de nature collective?}

Des travaux de recherche (Blanc-Maximin, Eymard, 2010) ont permis de réaliser une transposition de la notion de compétence collective issue du monde de l'entreprise (Le Boterf, 2000) dans le monde de l'enseignement primaire. Il s'avère que dans le monde de l'enseignement, deux compétences de nature collective existent (une compétence collective et une compétence du collectif) et se caractérisent par des indicateurs de sa présence (tableau 1). 
S'assurer une formation mutuelle

Dépasser les cloisonnements

Atteindre collectivement les résultats attendus

Mettre en place une charge équitable de travail

Agir en prenant et en mettant des décisions ayant fait l'objet de concertation
Faire partager sa compétence approfondie dans un domaine aux partenaires.

Se bâtir des représentations partagées sur les pédagogies, l'apprentissage, l'évaluation.

Echanger des idées, des « recettes », des artefacts pour éduquer au patrimoine.

Mettre en commun des documents didactiques et pédagogiques.

Réaliser les actions prévues dans le projet

Evaluer ensemble les effets du projet et réfléchir aux moyens d'évaluer les acquis des élèves

Répartir les charges de travail nécessaires à la réalisation du projet

Se donner des objectifs communs lors de la rédaction du projet. Corédiger le projet.

Tableau 1. Transposition d'indicateurs de la compétence collective pour un partenariat entre intervenants d'un PNR et enseignants.

Enfin, une synthèse opérée entre ces indicateurs (Blanc-Maximin, Eymard, 2010) et les travaux de Marcel et al. (2007) sur les verbes d'actions: coordonner, collaborer, coopérer va être utilisée pour repérer la nature des partenariats de type PNR-Éducation Nationale dans les cas que nous avons étudiés.

La compétence collective, proche de l'intelligence collective (Lévy, 1994), privilégie la résolution efficace du problème dans une visée de réactivité et de rentabilité pour l'organisation, le collectif et l'individu (Tableau 2). Projet d'une « intelligence partout distribuée, sans cesse valorisée, coordonnée en temps réel et qui aboutit à une mobilisation effective des compétences individuelles »(Lévy, 1994, p. 29), l'intelligence collective consiste à mobiliser au mieux et à mettre en synergie les compétences des individus, selon le principe que personne ne sait tout, que chacun sait quelque chose et que tout le savoir est contenu dans l'humanité. Des recoupements peuvent être établis avec un des objectifs de l'intelligence territoriale (Girardot et al., 2014) qui vise à proposer aux partenariats d'acteurs un processus cognitif et des outils de co-construction d'intelligence collective pour, avec, et par la communauté territoriale. Ce processus se concrétise dans le développement d'outils accessibles aux acteurs pour élaborer, gérer et évaluer des projets de développement durable. Elle se caractérise par un apprentissage professionnel de type hologrammatique (chacun est porteur du tout). 
Compétence collective : combinaison des compétences

individuelles des membres du collectif
Compétence du collectif : juxtaposition, coordination

des compétences individuelles des membres du

collectif

Coopération

Coordination et collaboration

Développer des compétences autres que celles individuelles: ce qu'on peut faire à plusieurs qu'on ne peut pas faire tout seul.

Combinaison, arrangement au sens mathématique

Collection au sens mathématique

Réunir en arrangeant dans un certain ordre en vue d'un certain résultat.

Synonyme d'un ensemble non-arrangé.

\section{Synergie}

Complémentarité de compétences spécifiques affirmées dans un domaine qui sont mobilisées de manière combinatoire pour résoudre un problème ou bâtir un projet, le réaliser.

Réalisation d'un apprentissage par les membres du collectif. Les uns apprennent aux autres et les autres apprennent aux uns : apprentissage collectif (formation mutuelle).

Apprentissage professionnel dans une dimension

hologrammatique (chacun a en lui tout ce qui a été décidé et appris).

Conservé en mémoire

Bénéfice cognitif pour les membres du projet

Créatrice de savoirs professionnels et de référence.

Elaboration de représentations partagées (Astier, 2006)

Image opératoire commune

Langage opératoire commun

Accepter l'existence de conflits en les gérant en temps opportun

\section{Coordination}

Pas de compétences affirmées mais l'association des compétences individuelles de chacun qui permet de réaliser un projet, de résoudre un problème.

Il existe probablement une forme des éléments appris. C'est dans la réalisation du produit ou du projet que se développe un apprentissage expérienciel.

Bénéfice pour l'action conduite mais pas forcément mise à distance, intégrée par les membres

Image opératoire pas forcément commune Langage opératoire commun

Eviter les conflits ou les laisser perdurer à l'état latent.

Tableau 2. Comparatif des caractéristiques des deux compétences.

La compétence du collectif se déploie dans la capacité des collectifs à mobiliser leurs ressources sans forcément ouvrir sur une construction de compétence pour chaque membre (tableau 2). C'est la qualité de l'action conduite (un projet par exemple) qui importe. Une porosité existe néanmoins entre ces deux compétences : l'apprentissage professionnel qui n'est pas un indicateur de la compétence du collectif peut intervenir ultérieurement chez les acteurs ; ces derniers ne sont pas obligés de développer une image opérative commune dans la compétence du collectif si le traitement ne correspond aux valeurs qui les agissent. Finalement, ce type de compétence collective (reliant les deux types) rend complémentaires une perspective organisationnelle et une approche valorisant l'ingéniosité des humains en accord avec leurs valeurs et conceptions professionnelles.

Il s'avère donc que la multiplication de projets éducatifs associant des acteurs d'un territoire à des enseignants pose des questions épistémologiques. La rencontre entre deux mondes éducatifs interroge les finalités, les valeurs et la forme scolaire. Elle questionne les professionnalités des acteurs et leur développement professionnel. Dans le cadre de ce texte, nous étudierons ce qui se joue lors de situations de pratiques partenariales entre acteurs «éducatifs » du territoire dans une perspective d'éducation au patrimoine. Plus précisément, nous cherchons à savoir dans quelle 
mesure et dans quelles conditions des pratiques partenariales entre intervenants d'un PNR et enseignants lors de projets d'éducation au patrimoine développent des compétences professionnelles de nature collective chez les acteurs.

\section{Trois études de cas en territoire rural labellisé}

Le contexte de l'étude s'inscrit dans un territoire rural isolé repéré pour ses difficultés socioéconomico-culturelles par le Ministère de l'Éducation Nationale. Il est labellisé Réseau d'Éducation Prioritaire en milieu rural. Les entrées culturelles patrimoniales sont ici privilégiées. Les acteurs éducatifs (enseignants et intervenants extérieurs sollicités par le PNR des Baronnies Provençales) ont pour projet d'engager les élèves dans la découverte d'éléments patrimoniaux ou potentiellement patrimoniaux, matériels et immatériels du territoire de vie dont les élèves et familles ne soupçonnent pas la richesse. La politique éducative des PNR sur le plan national vise à développer un ancrage territorial chez les élèves. Les enseignants ont pour visée l'acquisition de connaissances et compétences inscrites dans les programmes officiels en vigueur du Ministère de l'Éducation Nationale.

\subsection{Descriptifs des projets engagés dans les trois études de cas}

Cas A: L'éducation au patrimoine dans le développement du lien aux lieux patrimoniaux ou patrimonialisés de proximité

L'étude de cas A se déroule dans trois classes élémentaires multi-cours (du CP au CM2) situées dans trois villages ruraux de moins de 500 habitants et appartenant à un réseau d'éducation prioritaire. Le projet partenarial comprend l'intervention d'un photographe en résidence invité par le PNR. Le photographe souhaite relier les 3 écoles rurales dans un projet qui constituerait une forme de rupture d'un isolement géographique et surtout une valorisation des lieux patrimoniaux préférés de leur environnement. Chaque élève aura son portrait photographié par un de ses camarades dans le lieu du village qu'il considère comme bien commun à conserver pour les générations futures. Pour les plus jeunes élèves (de 5 à 7 ans), l'objectif visera à se faire photographier dans un lieu du village important pour soi, à faire découvrir par d'autres enfants du même âge. Par exemple, les élèves ont choisi des falaises (lieu d'escalade réputé au niveau européen), le bistrot, une ancienne loge datant de 1796, la rivière et son pont romain, un pré avec les moutons, un champ de lavandin, une tour sarrasine, mais aussi pour les très jeunes élèves la cabane et la fontaine de la place publique ou les jeux du jardin public de la commune.

\section{Cas B : Education au patrimoine local moyenâgeux}

Les trois classes de $5^{\circ}$ d'un collège classé en réseau d'éducation prioritaire rural et une association de valorisation du patrimoine sont impliquées. Le Moyen-Age constitue la thématique interdisciplinaire et plusieurs professeurs du collège (français, langues anciennes, arts plastiques, physique technologie, histoire participent au projet.

Le Parc Naturel Régional a désigné comme partenaire dans le projet une association loi 1901. Ses membres sont des personnes passionnées par le Moyen-âge et visent à valoriser, promouvoir le patrimoine médiéval à travers un campement pour faire découvrir les métiers et activités de cette époque au travers d'ateliers sur l'armement, l'escrime, l'archerie, les jeux d'adresse.

\section{Cas $C$ : Education au patrimoine naturel : les abeilles}

Ce projet concerne les deux classes d'école maternelle de moyenne et de grande section (élèves de 4-5 ans) d'une école rurale et un intervenant régulièrement sollicité par le PNR. Ce dernier propose des «activités pluridisciplinaires où le ludique, le scientifique, le sensoriel, le manuel et le visuel sont de mise » et souhaite apporter «connaissance et compréhension des concepts liés à la 
protection de la nature et à la préservation de l'environnement » (extraits de la page d'accueil du site internet de l'intervenant possédant le statut d'auto-entrepreneur). Ce projet a pour thème le patrimoine des abeilles comme un patrimoine et une biodiversité à protéger.

\subsection{Modalités de recueil des données}

Pour mener à bien nos études de cas et apporter des réponses à notre question de recherche, nous avons opté pour plusieurs outils méthodologiques. Une étude des projets déposés au service éducatif du PNR et à la circonscription de l'Education Nationale permet d'identifier la place du niveau de collaboration entre les partenaires dans la planification du projet.

L'observation dans les séances dédiées au projet et menées en partenariat vise à recueillir des informations sur le type de partenariat mis en œuvre devant les élèves. L'observation et la prise de notes durant les entretiens entre l'intervenant et l'enseignant-e (situés avant ou après le déroulement de la séance) s'intéressent à la qualité de la communication, de la négociation et de la contractualisation éventuelle. Les observations sont recueillies par des prises de notes ou par une captation vidéo des entretiens (si acceptation par les deux acteurs). Elles portent : sur la nature des contenus des échanges (coordination technique pratique des activités, organisation de cointervention durant les séances, partage anticipé des rôles de chacun, partage de documents ...), sur qui mène l'entretien (ascendant d'un acteur sur l'autre, répartition égale), sur les éventuels conflits et négociations, les mises au point entre les deux acteurs éducatifs et la manière de réguler l'avancée du projet. Elles permettent : l'analyse des pratiques pédagogiques observées des deux éducateurs durant les productions orales et photographiques des élèves et les modalités d'évaluation des acquis des élèves que l'un ou les deux mettent en œuvre pour nous renseigner sur la qualité du partenariat et les compétences professionnelles potentielles qui sont mobilisées.

\section{Résultats}

\subsection{L'étude des projets}

Cas A : L'aspect programmatique du projet du photographe en résidence impacte fortement l'écriture du projet final. Les intentions et objectifs des enseignantes convergent en fonction des propositions de l'intervenant. Les compétences que pourront travailler les élèves sont peu précisées et très peu déclinées en fonction des contenus des programmes officiels d'enseignement.

Cas B : Le projet «Bannières et blasons » est présenté comme un projet interdisciplinaire en lien avec la maîtrise de la langue. Le titre même du projet présenté par les enseignants indique une scission assez précise entre activités scolaires et interventions extérieures. Le projet comprend une partie principale rédigée par les professeurs d'une taille conséquente uniquement axée sur les contenus d'apprentissage (étude intégrale d'un ouvrage : Yvain ou le chevalier au lion ; étude de poésies d'amour courtois ; réalisation de blasons ; le fonctionnement des pont-levis, la maquette du château-fort; la société du Moyen-Age) traités dans chaque discipline scolaire autour de la thématique du Moyen Age. La perspective est pluridisciplinaire et non interdisciplinaire. Dans le projet, la place réservée aux objectifs et actions envisagés par les partenaires est succincte et présentée comme une mise en scène vivante de la vie au Moyen-Age dans un campement. Une fête médiévale (voir les détails au collège) est prévue pour clore le projet. Un autre volet présente un voyage de fin d'année sur le thème du Moyen âge à Paris. Une partition nette existe entre le scolaire et le «ludique », le «vivant » proposés par l'association de promotion du patrimoine médiéval. La forme scolaire (exercices, rédactions) durant les cours est tout à fait conservée et se juxtapose aux activités proposées dans le cadre patrimonial emblématique du château de la ville. Le projet se clôture par une grande fête médiévale en partie réservée aux élèves et en partie ouverte à tout public telle une animation pour le territoire local. La municipalité a fourni une aide matérielle conséquente. 
Cas C: La lecture du projet «Abeilles en conte, abeilles en danger » montre une réelle coécriture entre les attendus des enseignantes dont les objectifs et les compétences à acquérir sont très détaillés et conformes aux attendus des programmes officiels et ce que propose l'intervenant. La partie réservée à l'intervenant se révèle cohérente avec celle réservée à l'enseignant. Les compétences et les situations proposées sont complémentaires et identiques à celles décrites par les enseignantes. Un descriptif des séances indique la progression des apprentissages, les concepts travaillés, les co-interventions dans certaines séances et l'implication des familles d'élèves dans le projet.

\subsection{Les observations en classe}

Trois formes de partenariat sont identifiées. Certaines développent des pratiques de coordination, de collaboration ou de coopération et des indicateurs de la compétence du collectif ou de la compétence collective sont repérés. Ils sont potentiellement susceptibles de participer à un développement professionnel des acteurs.

\subsubsection{Cas $A$ : des pratiques partenariales différentes}

Ecole 1 : une confusion des rôles professionnels.

L'enseignante laisse l'intervenant faire cours à sa place sur des contenus qui pourtant lui incombent : le photographe joue par moments à l'enseignant, et l'enseignante l'autorise sans s'en préoccuper. Dans les temps de debriefing ou de préparation de la séance suivante, l'intervenant prend l'initiative des échanges et l'enseignante paraît acquiescer ses dires. Pas de conflits mais un consensus mou qui s'instaure. A partir de là, l'intervenant se montre relativement directif et devient réellement au fils du déroulement du projet le leader. Cette totale délégation du déroulement du projet à l'intervenant n'ouvre pas de perspectives à la mobilisation de compétences de nature collective.

\section{Ecole 2 : des conflits « positifs »}

L'enseignante veut conserver la main mise sur ce qui est proposé aux élèves dans le cadre de l'école, garder l'initiative, maintenir un contrôle de manière à ce que ses exigences soient respectées par l'intervenant. Elle veut conserver un pouvoir décisionnel sur ce qui va être fait et comment cela va être fait, notamment au niveau de l'évaluation des acquis des élèves. Elle n'entend pas se faire dicter ce qu'elle doit faire et exige que l'intervenant respecte scrupuleusement le cahier des charges du projet. Il en ressort des situations d'échanges conflictuels avec l'intervenant qui débouchent sur des avancées de part et d'autre. Le photographe a été un temps circonspect puis s'est rendu compte qu'il avait à y gagner car les exigences de sa partenaire comprenaient des pistes d'amélioration pour ses propres interventions.

\section{Ecole 3 : une absence de partenariat}

L'enseignante développe une attitude de retrait. Elle est réticente à ce projet qui a été quasiment imposé à cette école. Elle refuse tout entretien en dehors du temps de classe. Il n'y a pas de régulation entre partenaires et l'intervenant assure seul la conduite et la mise en œuvre du projet. Le partenariat se révèle inexistant et tout compétence de nature collective aussi.

\subsubsection{Cas B : une logique de "pré carré »}

Un partenariat de type cloisonné s'inscrit dans une logique de pré carré. Une coordination au niveau des aspects organisationnels est assurée mais rien n'est abordé en termes d'apprentissages cognitifs. Il semble que les actions de l'association sont là pour un faire-valoir du projet auprès des élus, des familles des élèves et des habitants du territoire. A ce titre, les discours des deux partenaires s'accordent pour organiser une fête médiévale. Une véritable partition entre savoirs 
scolaires et savoirs pratiques sur les techniques médiévales de combat et sur la gastronomie médiévale locale est installée. Les échanges des partenaires portent sur l'organisation de la fête (conditions de sécurité, autorisations municipales, invitations...).

\subsubsection{Cas $C$ : un partenariat vecteur de compétences de nature collective}

Les indicateurs d'une compétence collective sont repérés. Les partenaires partagent des savoirs de référence, des pratiques pédagogiques et se nourrissent de l'expérience des uns et des autres. Ils ont une image opérative commune et échangent régulièrement en utilisant un langage commun. Nous notons la présence de temps d'analyse réflexive et de régulation systématiques avant et après chaque séance du projet. Le projet initial est systématiquement affiné en fonction des avancées des élèves et, au fur et à mesure de l'avancement du projet, les partenaires s'approprient un langage commun. Les échanges pédagogiques et didactiques sont nombreux et s'appuient sur le partage de documents, l'échange de matériels pédagogiques, la recherche de créativité dans les situations proposées aux élèves. Les enseignantes montrent à l'intervenant les travaux réalisés en classe à partir de la séance précédente et lui en indiquent les avancées. Par exemple, l'intervenant utilise une formulette sur les abeilles apprise en classe pour un temps de transition dans la séance.

Chaque enseignante s'accorde avec l'intervenant sur leurs modalités de co-intervention. Par exemple, une enseignante va pouvoir se mettre en retrait durant 20 minutes pour observer les attitudes et l'activité de deux élèves hyperactifs et d'un élève habituellement inhibé. Avant la séance, l'intervenant a sollicité l'enseignante et l'ATSEM pour effectuer des tâches durant la séance.

\subsection{Présence de compétence collective et de compétence du collectif?}

Le cas A école 1 présente quelques rares indicateurs de la présence d'une compétence du collectif. Il existe un bénéfice pour l'action conduite mais celle-ci n'est pas forcément mise à distance, soumise à réflexion et intégrée par les acteurs. Il en résulte une image opératoire qui n'est pas forcément commune. L'observation des temps de classe et des entretiens montre qu'un des objectifs de l'enseignante est d'éviter tout conflit. Celle-ci n'oppose aucun refus aux propositions du photographe et ne fait pas preuve d'initiative, n'est pas force de proposition. Elle participe, dans l'intérêt d'une réalisation « sans vague » du projet, en respectant scrupuleusement les directives de l'intervenant. Une réflexion conjointe sur les actions menées n'existe pas. Par conséquent, l'image opératoire commune est très floue dans les dires de l'enseignante auprès des élèves.

Le cas A école 2 offre cinq indicateurs de la présence d'une compétence du collectif et cinq indicateurs de la présence d'une compétence collective. Il donne à voir le développement de compétences autres que celles individuelles. Les acteurs identifient ce qu'on peut faire à plusieurs qu'on ne peut pas faire tout seul. Il procède par combinaison, arrangement au sens mathématique (réunir les idées, les propositions des acteurs en arrangeant dans un certain ordre, en vue d'un certain résultat). Des compétences sont mises en synergie au travers d'un langage opératoire commun. Des conflits existent et ils sont gérés en temps opportun. Il existe probablement une forme des éléments appris chez les deux types d'acteurs. Ce partenariat occasionne la mise en synergie des compétences des deux intervenants et engage l'emploi d'un langage opératoire commun. C'est dans la réalisation du produit ou du projet que se développe un apprentissage expérientiel.

Le rôle des conflits est un élément remarquable car leur gestion en temps opportun débouche sur une amélioration des situations et pratiques pédagogiques en direction des élèves. Grâce aux interactions conflictuelles des partenaires-chacun voulant s'arroger des prorogatives ou les conserver-, la mise en synergie des compétences de chacun, par exemple en matière d'évaluation des acquis des élèves, a bénéficié au niveau de l'action conduite. Les partenaires ont mis en commun leurs connaissances, savoir-faire et attitudes respectifs dans le cadre d'une démarche d'évaluation critériée co-construite avec les élèves. Cette démarche est la résultante d'une 
combinaison de compétences des partenaires. La compétence de nature collective affirmée est cependant réduite à cet exemple. Il n'y a pas eu d'évaluation finale du projet au niveau de la qualité de l'efficacité et de l'intérêt d'un partenariat. L'image opératoire commune s'est construite pas à pas durant le déroulement du projet mais l'association des compétences individuelles de chacun a permis de réaliser un projet, de résoudre un problème.

Le cas A école 3 représente un cas de quasi- absence de partenariat. Le seul indicateur repéré est la volonté de ne pas créer de conflits grâce à un laisser-faire total de l'intervenant durant ses interventions en classe qui n'ont été ni préparées conjointement, ni régulées conjointement avec l'enseignante. Il n'a pas été question, pour les partenaires, d'évaluer cette action.

Le cas B offre un grand nombre d'indicateurs de la présence de la compétence du collectif. La coordination et la collaboration sont limitées au positionnement de l'ordre des temps du projet et surtout à l'organisation de la fête médiévale qui, d'une part, clôturait le projet et donnait à voir la complémentarité des acteurs éducatifs du projet et, d'autre part, constituait une vitrine pour l'association intervenante. Il apparaît clairement que les deux parties ont dû développer des compétences autres que celles individuelles : ce qu'on peut faire à plusieurs qu'on ne peut pas faire tout seul. Par exemple, les compétences d'organisation d'une telle manifestation sont particulièrement mobilisées : une professeure déléguée par ses collègues a bâti le programme qu'elle a soumis au responsable de l'association. Il s'agit de réaliser une collecte de compétences qui seront coordonnées. C'est l'association des compétences individuelles de chacun qui permet de réaliser un projet ou de résoudre un problème. Le bénéfice visé est uniquement orienté sur la réussite de l'action conduite. Les échanges auxquels nous avons assisté ne portent absolument pas sur la teneur des apprentissages proposés par les uns et les autres ou sur les modalités pédagogiques utilisées. Cette mobilisation de compétences diverses n'est ni mise à distance, ni intégrée par les membres qui ne souhaitent pas partager leurs connaissances et leurs savoir-faire. Les intervenants sont identifiés par les professeurs comme des prestataires de services. Les propos recueillis durant les échanges, qui précèdent les interventions en classe ou lors de la fête médiévale, montrent seulement une complémentarité «pour permettre aux élèves de découvrir la littérature du Moyen-Age et de se mettre de manière ludique dans la peau d'un chevalier » déclare la professeure de langues anciennes. La professeure de français complète le propos de sa collègue : «nous travaillons sur l'histoire d'un chevalier donc c'est très bien que vous les fassiez utiliser des armes, observer ou participer à des combats. Chacun pourra comprendre, cela donnera du sens ».

Le cas $\mathrm{C}$ présente tous les indicateurs de la présence d'une compétence collective. La coopération existe à tous les stades du projet avec un partage de connaissances (les types d'insectes butineurs, la pollinisation des fleurs par les abeilles, la vie dans une ruche et les rôles de différentes abeilles, les dangers qui les guettent), un échange de modalités pédagogiques (l'intervenant a conçu des artefacts- jeux et petit film d'animation- adaptés aux jeunes élèves et l'enseignante a recensé des contes sur les abeilles), des échanges de pratiques (pour reconnaître les parties du corps d'une abeille en produisant celui-ci en land-art pour l'intervenant, en utilisant une peluche puis des découpages et collages des différentes parties du corps de l'abeille et enfin en faisant dessiner une abeille pour l'enseignante). Un apprentissage professionnel est réalisé par les membres du collectif (les deux enseignantes et l'intervenant). Les uns apprennent aux autres et les autres apprennent aux uns dans une formation mutuelle de type informel. Une dimension hologrammatique (chacun a en lui tout ce qui a été décidé et appris) est présente. Lors d'un entretien entre les partenaires avant une séance, l'enseignante a expliqué qu'elle avait essayé de reproduire une partie de la séance de l'intervenant auprès des élèves qui avaient été absents et qu'elle y était parvenue. L'intervenant a répondu : « c'est amusant, j'ai intégré un extrait du conte que tu m'as fait découvrir dans une classe de cours élémentaire $2^{\mathrm{e}}$ année et $\mathrm{j}$ 'ai pu créer une entrée pour faire réfléchir à la perte de biodiversité ». Une co-intervention est négociée et programmée dans chaque séance où l'intervenant est présent (s'organiser dans un certain ordre en vue d'obtenir un certain résultat). Par exemple, l'intervenant et chaque enseignante ont réfléchi ensemble à des moyens d'évaluer les progrès des 
élèves de maternelle. Chacun essaie de trouver des situations d'évaluation et l'enseignante a la mission (qui correspond aussi à ses prérogatives) de récolter des traces des acquis. Ce ne sont pas seulement des connaissances ou des capacités qui sont repérées. Une décision commune a été prise concernant le rôle de chacun. Lors d'une séance menée par l'intervenant, une enseignante s'est intéressée exclusivement aux comportements de quatre enfants qui, dans la classe, n'arrivaient pas encore à adopter des attitudes d'élève illustrant le fait de développer des compétences autres que celles individuelles : ce qu'on peut faire à plusieurs qu'on ne peut pas faire tout seul. Les deux acteurs ont échangé sur les résultats de cette observation et sur la manière d'intéresser et de canaliser ces élèves.

Pour synthétiser, les indicateurs de la présence d'une compétence collective concernant le cas C sont nombreux. Dans le cas A école 2, les indicateurs repérés dénotent nettement la présence d'une compétence du collectif et certains d'entre eux s'inscrivent dans le cadre d'une compétence collective. Des traces d'une compétence du collectif existent dans le cas B. Quant aux cas A école 1 et A école 3, rien ne prouve l'existence de ce type de compétences. Ces études de cas issus d'un même partenariat institutionnel identifient des niveaux de partenariat très différents qui offrent ou non des opportunités de développement professionnel. Les obstacles repérés se situent au niveau des visées des acteurs éducatifs. Certains n'ambitionnent que la réussite de l'action au détriment d'éventuelles sources d'apprentissages professionnels que sa conception et sa réalisation permettraient. D'autres s'inscrivent dans une visée consumériste des interventions extérieures réalisées dans leurs classes et ne s'impliquent pas ou peu pour acquérir un peu plus d'expertise. Enfin, certains enseignants et un intervenant se saisissent de l'opportunité des situations dans ce type de projet (construction, réalisation, temps réflexifs intermédiaires ...).

\section{Conclusion}

Pour répondre à notre question sur les conditions favorables au développent des compétences professionnelles de nature collective chez les acteurs lors de pratiques partenariales entre intervenants d'un PNR et enseignants lors de projets d'éducation au patrimoine, nous avons étudié quelques cas en milieu rural sur une aire protégée. Il en ressort les éléments suivants.

Pour qu'un partenariat entre éducation et apprentissage formels et non formels s'avère riche, la partition des acteurs devrait, a minima, être clairement identifiée : mettre au jour et assumer les visées éducatives et éthiques de chaque partenaire, agir en fonction des compétences, en la matière, de chacun des partenaires et tenir compte des objectifs institutionnels prescrits aux professeurs des écoles. Un travail de formation professionnelle et associative au partenariat est à engager conjointement pour acquérir et mobiliser des compétences de coopération et de collaboration entre acteurs d'un projet éducatif scolaire car les actions d'éducation au patrimoine se situent nécessairement dans des temporalités longues que seul un partenariat entre structures formelles et informelles peut assumer. Les pratiques partenariales dans des projets d'éducation au patrimoine et dans leur mise en œuvre sont très variées et probablement la variété de leur efficience peut impacter les apprentissages des élèves et des acteurs éducatifs. Il n'est pas question bien sûr d'uniformiser les pratiques des partenaires mais de leur permettre d'apprendre professionnellement à travailler ensemble.

Un obstacle de taille s'inscrit dans la conception du partenariat de l'école française pensée dans une perspective d'assistance (l'intervenant extérieur est un expert qui vient au secours de l'enseignant) et de subordination (l'intervenant ne représente qu'une aide ponctuelle pour l'enseignant). La relation entre partenaires est alors hiérarchisée : les missions propres de l'école prévalent sur les missions qu'elle n'arrive pas à assumer seule. Même si le rapport Thélot stipule que seule l'école éduque et que les autres acteurs éducatifs développent la socialisation, les cas que nous avons étudiés démentent cette affirmation. $\mathrm{Si}$, institutionnellement, les partenaires sont considérés comme des experts, avec un statut d'assistanat du professeur dans une relation de 
subordination, trop souvent encore, des projets éducatifs sont élaborés avec des partenaires, mais pas entre partenaires.

La question de l'évolution de cette situation pour la réussite des élèves et pour l'acquisition de nouvelles compétences professionnelles chez les acteurs éducatifs est posée. Nous plaidons pour des temps de formation commune de ces derniers sur un territoire qui en serait à la fois le mobile et le promoteur. Cette piste constituerait certes un cadre innovant de formation pour l'Éducation nationale et le partenaire local, étant donnée sa dimension contextualisante. Est-ce que les territoires labellisés «aires protégées» pourraient, par leurs caractéristiques, être des instigateurs de formations d'un nouveau type validées par l'Éducation nationale?

La loi de refondation pour l'École de la République (2013) souhaitait faire cheminer les élèves à travers des parcours artistiques et culturels, avenir, santé et citoyen, options non remises en question dans la loi de 2019. Les orientations de ces deux dernières lois ont fait émerger une multiplicité des « éducations à », signe d'une école à laquelle la société confie ou délègue la gestion de certains de ses problèmes contemporains. Par ailleurs, on assiste à un mouvement de balancier entre une école située sciemment hors de la vie pour se concentrer sur des apprentissages scolaires et protéger les enfants des dangers (Vincent, 1994 ; Levrard \& Delalande, 2015) et une école « contextualisante » prenant en compte le contexte local, environnemental, ouverte sur les réalités locales qui considère le territoire comme un support digne d'apprentissages. Ces éducations contribuent à concevoir l'école comme une expérience singulière du monde qui se vit dans une expérience collective et partagée. Dans ces conditions, le collectif d'acteurs éducatifs d'un territoire devrait pouvoir, au lieu de continuer d'agir dans des conditions de subordination et d'assistance, s'orienter vers des perspectives d'intelligence collective (Lévy, 1994) et d'intelligence territoriale (Girardot \& al., 2014).

\section{Bibliographie}

Ardoino, J. (1993). L'approche multiréférentielle en formation et en sciences de l'éducation, Formation Permanente, 25-26, Paris : Université Paris VIII.

Astier, P. (2006). Apprendre et agir « ensemble ». Colloque SIFA-CREAD. Rennes 23 et 24 juin.

Barthes, A., Alpe, Y. \& Blanc-Maximin, S. (2015). L'éducation au patrimoine, un outil pour le développement local ? Positionnement critique vers l'instrumentalisation de l'éducation au service de la labellisation des territoires ? Revue francophone du Développement Durable Hors- Série 3, p. 8-22. Clermont-Ferrand: éditions Économia.

Berryman, T. (2003). L'éco-ontogenèse : les relations à l'environnement dans le développement humain - d'autres rapports au monde pour d'autres développements. Éducation relative à l'environnement : Regards - Recherches Réflexions, $n^{\circ} 4$, p. 207-228.

Blanc S., Eymard C. (2010). Compétences d’un collectif d'école. Questions Vives, n5, p. 43-55.

Boutinet, R. (2005). Anthropologie du projet. Paris : Presses universitaires de France.

Charlot, B. (1997). Du rapport au savoir, éléments pour une théorie. Paris, Anthropos.

Charte des Parcs Naturels Régionaux [En ligne]: https://www.data.gouv.fr/fr/datasets/parcs-naturels-regionaux-de-laregion-sud/

Clanet, C. (1993). L'interculturel : introduction aux approches interculturelles en éducation et en sciences humaines. Toulouse: P.U. du Mirail.

Clot, Y. (2007). Psychopathologie du travail clinique. [En ligne] : www.comprendre-agir.org/images/fichierdyn/doc/2007/psychopathologie-travail-clinique-activite-yves-clot-chsct.pdf

Devos-Prieur Odile, Loubet-Gauthier Elisabeth (2002. Incidence des modes de partenariat à l'école élémentaire sur la polyvalence des maîtres et sur les représentations des élèves. Recherche \& Formation, Les dynamiques identitaires : questions pour la recherche et la formation, $\mathrm{n}^{\circ} 41$. p. 153-170.

Dubois M., Retour, D. (1999). La compétence collective : validation empirique fondées sur les représentations opératoires de travail partagées. Psychologie du travail et des organisations, Vol. 5, n²-1, p. 225-243. 
Dupont, N. (2010). Les partenariats écoles / institutions culturelles des passages entre cultures juvéniles, cultures artistiques et cultures scolaires? Les Sciences de l'éducation -Pour l'Ère nouvelle/4, Vol. n³ p.95-121.

Eneau,J. (2016).Autoformation, autonomisation et émancipation. De quelques problématiques en formation d'adultes. Recherches \& éducations, 21-38.

Fourez, G. (1998). Se représenter et mettre en oeuvre l'interdisciplinarité à l'école. Revue des sciences de l'éducation, n²4 (1), 31-50. https://doi.org/10.7202/031960ar

Garnier, B. (dir.), (2014). Politiques d'éducation et identités territoriales. Carrefours de l'éducation, n³8, décembre.

Khalali, F., Therriault, G., et Bader, B. (2019). "Rapport aux savoirs d'élèves du secondaire en lien avec l'environnement et le développement durable en France et au Québec : rapport épistémique et contextualisé au monde ». », Éducation et socialisation [En ligne], 51 | 2019, mis en ligne le 31 mars 2019, consulté le 23 avril 2019. URL: http://journals.openedition.org/edso/5693; DOI: 10.4000/ edso.5693

Girardot, J.-J. (2014). L'observation territoriale coopérative et participative catalyseur de l'intelligence territoriale. Repéré à : https://slideplayer.fr/slide/1289485.

Lange, J-M. (2017). «Éducations à », territoires et postures des enseignants : ruptures et tensions. In A. Barthes, P. Champollion, Y. Alpe (dir.). Permanence et évolutions des relations complexes entre éducations et territoires. Volume 1, p.81-92. Londres: ISTE.

Le Boterf, G. (2000). Construire les compétences individuelles et les compétences collectives. Paris: Editions d'Organisation.

Levrard, S. \& Delalande, J. (2015). «Mon école, c'est pas que je la trouve belle, c'est qu'on est ensemble », Diversité, $\mathrm{n}^{\circ} 179$, pp.131-135. Communications Expand. 20 - 21 juin 2019.

Lévy, P. (1994). L’intelligence collective ; pour une anthropologie du cyberspace. Paris: Éditions de la Découverte.

Marcel, J.-F., Dupriez, V., Périsset Bagnoud, D., Tardif, M. (2007). Coordonner, collaborer, coopérer. De nouvelles pratiques enseignantes. Bruxelles: De Boeck Supérieur.

Maulini, O., Perrenoud, P. (2005). La forme scolaire de l'éducation de base : tensions internes et évolutions. In O. Maulini, C. Montandon (coord.), Les formes de l'éducation : variété et variations. Bruxelles: De Boeck Supérieur.

MEN (1992). Circulaire $\mathrm{n}^{\circ} 92-196$ du 3 juillet 1992 : participation d'intervenants extérieurs aux activités d'enseignement dans les écoles maternelles et élémentaires. Paris.

MENESR (2013). Loi d'orientation et de programmation pour la refondation de l'École de la République du 8 juillet 2013. Paris, France. Récupéré du site : http://www.education.gouv.fr /cid2770/le-socle-commun-de-connaissanceset-decompetences.html.

MENESR (2013). Référentiel des compétences des métiers du professorat et de l'éducation. B.O.E.N. n 30 du 25 juillet.

Mérini, C. (2006). Nature et limites des partenariats éducatifs. ADBD P. [consultable en ligne, URL: http://adbdp.web03.b2f-concept.net/spip.php?article421].

Mérini, C. (2007). La dimension organisationnelle du travail enseignant: déterminant de l'ancrage de l'ES dans les apprentissages des élèves. Premier colloque de la chaire en éducation à la santé du Canada. Université du Québec à Montréal, Montréal, Janvier. 〈hal-01396336〉

Mérini,C., Bizzoni-Prévieux, C. (2007). Le travail partagé: exploration en formation des maitres. Congrès international AREF. Actualité de la Recherche en Education et Formation du 29 août-1 septembre. Strasbourg.

Schwartz, Y., Durrive, L. (coord., 2003). Travail \& Ergologie, Entretiens sur l'activité humaine. Toulouse: Octarès Éditions.

Thélot, C. (2004). Pour la réussite de tous les élèves, Rapport de la commission du débat national sur l'avenir de l'école présidée par Claude Thélot. Paris: SCÉRÉN/CNDP, La Documentation française.

Thomazet, S., Mérini, C. (2014). Le travail collectif, outil d’une école inclusive? Questions Vives, n²1 [En ligne URL: http:// questionsvives.revues.org/1509; DOI: 10.4000/questionsvives.1509].

Vincent, G. (coord., 1994). L'éducation prisonnière de la forme scolaire? Scolarisation et socialisation dans les sociétés industrielles. Lyon: Presses universitaires de Lyon.

Wittorski, R. (1997). Analyse du travail et production de compétences collectives. Paris: L’Harmattan, collection « Action et Savoir». 
Zarifian, P. (1999). Objectif compétence. Pour une nouvelle logique. Paris: éditions Liaisons.

Zay, D. (coord., 1994). Enseignants et partenaires de l'école. Démarches et instruments pour travailler ensemble. Recherche \& Formation, ${ }^{\circ} 17$, p. 166-169.

\section{Annexe}

\begin{tabular}{|c|c|}
\hline $\begin{array}{l}\text { Compétence collective : combinaison des } \\
\text { compétences individuelles des membres } \\
\text { du collectif }\end{array}$ & $\begin{array}{l}\text { Compétence du collectif : juxtaposition, coordination des } \\
\text { compétences individuelles des membres du collectif }\end{array}$ \\
\hline Coopération Cas C & Coordination et collaboration Cas $\mathrm{A} 2$ et $\mathrm{B}$ \\
\hline
\end{tabular}
tout seul. Cas A2, B et C

Combinaison, arrangement au sens mathématique. Réunir en arrangeant dans un certain ordre en vue d'un certain résultat. Cas $\mathrm{A} 2$ et $\mathrm{C}$

\section{Synergie Cas A2 et C}

Complémentarité de compétences spécifiques affirmées dans un domaine qui sont mobilisées de manière combinatoire pour résoudre un problème ou bâtir un projet, le réaliser. Cas C

Réalisation d'un apprentissage par les membres du collectif. Les uns apprennent aux autres et les autres apprennent aux uns: apprentissage collectif (formation mutuelle).

Apprentissage professionnel dans une dimension hologrammatique (chacun a en lui tout ce qui a été décidé et appris).

Cas C

Conservé en mémoire

Bénéfice cognitif pour les membres du projet

Créatrice de savoirs professionnels et de référence. Cas $\mathrm{C}$

Elaboration de représentations partagées (Astier, 2006) Cas C

Image opératoire commune Cas $\mathrm{C}$

Langage opératoire commun Cas $\mathrm{A} 2$ et $\mathrm{C}$

Accepter l'existence de conflits en les gérant en temps opportun Cas A2
Collection au sens mathématique

Synonyme d'un ensemble non arrangé. Cas B

\section{Coordination Cas B}

Pas de compétences affirmées mais l'association des compétences individuelles de chacun qui permet de réaliser un projet, de résoudre un problème. Cas $\mathrm{A} 2$ et $\mathrm{B}$

Il existe probablement une forme des éléments appris. C'est dans la réalisation du produit ou du projet que se développe un apprentissage expérienciel. Cas A2

Bénéfice pour l'action conduite mais pas forcément mise à distance, intégrée par les membres.

Cas A1, A2, B

Image opératoire pas forcément commune Cas A1, A2, B

Langage opératoire commun Cas A2

Eviter les conflits ou les laisser perdurer à l'état latent. Cas A1, A3 